\title{
Tunneling magnetic effect in heterostructures with paramagnetic impurities
}

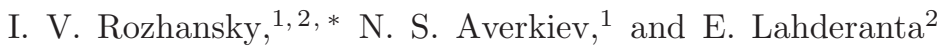 \\ ${ }^{1}$ A.F. Ioffe Physical Technical Institute, Russian Academy of Sciences, 194021 St.Petersburg, Russia \\ ${ }^{2}$ Lappeenranta University of Technology, P.O. Box 20, FI-53851, Lappeenranta, Finland
}

(Dated: August 12, 2018)

\begin{abstract}
An effect of paramagnetic impurity located in a vicinity of a quantum well (QW) on spin polarization of the carriers in the QW is analyzed theoretically. Within approach of Bardeen's tunneling Hamiltonian the problem is formulated in terms of Anderson-Fano model of configuration interaction between a localized hole state at Mn and continuum of heavy hole states in the InGaAs-based QW. The hybridization between the localized state and the QW leads to resonant enhancement of interband radiative recombination. The splitting of the configuration resonances induced by splitting of the localized state in magnetic field results in circular polarization of light emitted from the QW. The developed theory is capable of explaining known experimental results and allows for calculation of the photoluminescence spectra and dependence of integral polarization on temperature and other parameters.

PACS numbers: 75.75.-c, 78.55.Cr, 78.67.De
\end{abstract}

\section{INTRODUCTION}

Various phenomena based on interference of a discrete quantum-mechanical state and a continuum of states have

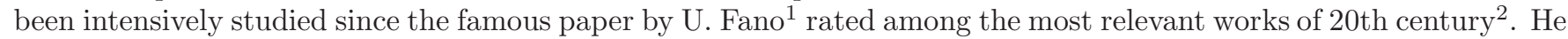
suggested a theoretical approach often regarded as Fano-Anderson model or Fano configuration interaction which was successfully applied to explain the characteristic asymmetric resonances observed in atomic spectroscopy experiments. It further appeared that numerous examples of Fano resonances existed in atomic and nuclear physics, condensed matter physics and optics 2 . The co-existence of a localized discrete level and continuum of states within the same energy range is also quite common in low-dimensional semiconductor structures ${ }^{2}-\underline{5}$. The structures containing a quantum well $(\mathrm{QW})$ and a ferromagnetic or paramagnetic layer located in the vicinity of the QW nowadays are gaining a special interest as they are believed to combine the high mobility of the carriers in the QW and magnetic properties provided by the magnetic layer. In particular, an exchange interaction with ferromagnetic layer leads to spin polarization of holes ${ }^{6}$. For GaAs-based structures with a $\delta$-layer of Mn the holes probably play an important role in promoting ferromagnetic state of the Mn layer ${ }^{7.8}$. The system considered in the present work consists of GaAsbased heterostructure with a $\operatorname{In}_{x} \mathrm{Ga}_{1-x}$ As QW $(x=0.1-0.2)$ and $\delta$-layer of paramagnetic acceptor $(\mathrm{Mn})$ located at a distance of a few nanometers from the QW. A number of recent experiments show that the Mn $\delta$-layer gives rise to circular polarization of the photoluminescence from the QW in magnetic field ${ }^{9}, 10$. It was found that the intensities of the radiation at the wavelength corresponding to interband direct transitions in the QW differ for opposite circular polarizations in magnetic field applied normally to the QW plane. If $\mathrm{Mn}$ is replaced by a non-magnetic acceptor (carbon) no polarization is registered. Thus the observed polarization is not due to g-factor of the free carriers in the QW which in this way is proved to be small. On the contrary, the holes localized at Mn do have $g \approx 3 \frac{11}{}$ and possibly can penetrate into the QW by means of quantum-mechanical tunneling which is expected to be of resonant-type if the energy of the localized state coincides with that of free 2D hole in the QW. We use the Fano configuration interaction approach to study tunneling hybridization between a localized hole state at paramagnetic impurity with the 2D continuum of states in the QW. We show how this hybridization should reveal itself in the photoluminescence at the QW wavelength. The theory allows for calculation of circular polarization degree in magnetic field. We have to mention here that there are other mechanisms that might contribute to the observed polarization like non-resonant tunneling of electrons from QW to Mn and recombination with the holes localized at Mn. While it still remains unclear which of the mechanisms has larger contribution to the polarization in experiments on photolumenescence ${ }^{9.10}$, in our paper we restrict our consideration by configuration interaction for the holes quite naturally expected in the p-type system under study.

\section{HOLE STATES AT ACCEPTOR AND IN QUANTUM WELL}

We assume that the eigenstates of the holes in the QW form a continuum of states starting from the energy of size quantization (taken for zero of energy scale). These 2D states can be characterized by two quantum numbers, say $k_{x}$ and $k_{y}$ are the projections of $2 \mathrm{D}$ wavevector on the axis $x$ and $y$ in the plane of the QW. Considering interaction with 
a point-like impurity it is more convenient use cylindrical basis. In this case each state in the QW is characterized by the magnitude of the wavevector $k$ and index $l$ denoting the cylindrical harmonic. The corresponding wavefunction is given by:

$$
\varphi_{k l}(\rho, z)=\eta(z) \frac{\pi^{1 / 4}}{\sqrt{2}} \frac{\sqrt{k}}{S^{1 / 4}} J_{l}(k \rho) e^{i l \theta},
$$

where $J_{l}(k \rho)$ is Bessel function of order $l, \rho$ and $\theta$ are the polar coordinates in the plane of the QW, $S$ is the area of the QW plane, $\eta(z)$ is the envelope function of size quantization in $z$-direction. The wavefunction is normalized to unity. Firstly, let us treat this continuum as a set of discrete states characterized by energies $\varepsilon_{k}$ and wavefunctions $\varphi_{k l}$. Considering the free $2 \mathrm{D}$ carriers implies the magnetic field applied to the $\mathrm{QW}$ to be nonquantizing. The validity of this assumption is discussed in section VI Below we consider $\operatorname{In}_{x} \mathrm{Ga}_{1-x}$ As QW having only one level of size quantization for the heavy holes, we neglect the light holes being split off due to size quantization. Thus the basis of Bloch amplitudes to be used is formed of the states with certain projection of total angular momentum $J=3 / 2$ on $z$ axis which is perpendicular to the QW plane:

$$
\left(e_{3 / 2}, e_{1 / 2}, e_{-1 / 2}, e_{-3 / 2}\right) .
$$

The wavefunctions $\varphi_{k l, j}$ in this basis have the form:

$$
\varphi_{k l,-\frac{3}{2}}=\left(\begin{array}{c}
0 \\
0 \\
0 \\
\varphi_{k l}(\rho)
\end{array}\right), \quad \varphi_{k l,+\frac{3}{2}}=\left(\begin{array}{c}
\varphi_{k l}(\rho) \\
0 \\
0 \\
0
\end{array}\right) .
$$

The kinetic energy of a state in the QW is related to the wavevector value as

$$
\varepsilon=\frac{\hbar^{2} k^{2}}{2 m_{h h}^{\prime}},
$$

where $m_{h h}^{\prime}$ is the in-plane heavy hole mass in the QW.

In order to determine wavefunction $\psi$ of a hole localized at an acceptor one should consider the kinetic part of the Luttinger Hamiltonian and attractive potential of the acceptor $U(r)$. The spherically symmetrical potential preserves the symmetry $\Gamma_{8}$, thus the ground state is 4 -fold degenerate and can be classified by projection of angular momentum. The eigenfunctions of Luttinger Hamiltonian with spherically symmetric attractive potential can be explicitly found in the model of zero radius potential12. In the basis of Bloch amplitudes they are expressed as follows:

$$
\begin{gathered}
\psi_{+\frac{3}{2}}=\left(\begin{array}{c}
R_{0} Y_{00}+\frac{1}{\sqrt{5}} R_{2} Y_{20} \\
-\frac{2}{\sqrt{10}} R_{2} Y_{21} \\
\frac{2}{\sqrt{10}} R_{2} Y_{22} \\
0
\end{array}\right), \psi_{+\frac{1}{2}}=\left(\begin{array}{c}
\frac{2}{\sqrt{10}} R_{2} Y_{2,-1} \\
R_{0} Y_{00}-\frac{1}{\sqrt{5}} R_{2} Y_{20} \\
0 \\
\frac{2}{\sqrt{10}} R_{2} Y_{22}
\end{array}\right), \\
\psi_{-\frac{1}{2}}=\left(\begin{array}{c}
\frac{2}{\sqrt{10}} R_{2} Y_{2,-2} \\
0 \\
R_{0} Y_{00}-\frac{1}{\sqrt{5}} R_{2} Y_{20} \\
\frac{2}{\sqrt{10}} R_{2} Y_{21}
\end{array}\right), \psi_{-\frac{3}{2}}=\left(\begin{array}{c}
\frac{1}{\sqrt{5}} R_{2} Y_{2,-2} \\
-\frac{2}{\sqrt{10}} R_{2} Y_{2,-1} \\
R_{0} Y_{00}+\frac{1}{\sqrt{5}} R_{2} Y_{20}
\end{array}\right) .
\end{gathered}
$$

Here

$$
\begin{aligned}
R_{0} & =C_{0}\left(\frac{\beta}{r} e^{-q r \sqrt{\beta}}+\frac{e^{-q r}}{r}\right) \\
R_{2} & =C_{0}\left(\frac{\beta}{r} e^{-q r \sqrt{\beta}}\left(1+\frac{3}{q r \sqrt{\beta}}+\frac{3}{q^{2} r^{2} \beta}\right)-\frac{e^{-q r}}{r}\left(1+\frac{3}{q r}+\frac{3}{q^{2} r^{2}}\right)\right), \\
C_{0} & =\sqrt{\frac{q}{\beta^{3 / 2}+1}}, \\
q & =\sqrt{\frac{2 m_{h h} E_{0}}{\hbar^{2}}} \\
\beta & =\frac{m_{l h}}{m_{h h}},
\end{aligned}
$$


$E_{0}$ is the binding energy of a hole at the acceptor, $Y_{l m}$ are the spherical harmonics. $m_{l h}, m_{h h}$ - respectively are the bulk masses of light hole and heavy hole in GaAs. Note, that the radial part of all nonzero components of the wavefunctions (4) have two characteristic decay lengths, the largest of the two being always determined by the light hole mass $m_{l h}$.

\section{TUNNELING BETWEEN ACCEPTOR AND QUANTUM WELL FOR COMPLEX BAND STRUCTURE}

For the studied system the potential barrier separating the Mn $\delta$-layer from the QW is weakly transparent for tunneling so the localized hole state mix up with the QW states. Rigorous calculation of eigenfunctions of the resulting state is rather hard to perform as it requires solving stationary Schrodinger equation in the complicated 3D potential and with account for the complex valence band structure. In order to circumvent the explicit solving of Schrodinger equation for tunneling problems the so-called tunneling or transfer Hamiltonian formalism is commonly used as originally proposed by Bardeen 13 . Within this approach the total tunneling Hamiltonian is expressed as $H=H_{M n}+H_{Q W}+H_{T}$, where $H_{M n}$ is partial Hamiltonian having the localized hole state at Mn as its eigen state. $H_{Q W}$ in the same way corresponds to the QW itself, its eigenfunctions $\varphi_{k}$ form non-degenerate continuum of states characterized by quantum number $k$. The term $H_{T}$ accounts for the tunneling. In the secondary quantization representation the total Hamiltonian can be written as follows:

$$
H=\varepsilon_{0} a^{+} a+\sum_{k} \varepsilon_{k} c_{k}^{+} c_{k}+\sum_{k} T_{k} c_{k}^{+} a+T_{k}^{*} a^{+} c_{k},
$$

where $a^{+}, a$ - the creation and annihilation operators for the localized state characterized by its energy $\varepsilon_{0}$, and $c_{k}^{+}, c_{k}$ - the creation and annihilation operators for the continuum state having energy $\varepsilon_{k}$. For the convenience both energies $\varepsilon_{0}$ and $\varepsilon_{k}$ here and below are measured from the level of size quantization of holes in the QW so that $\varepsilon_{k}$ is simply their kinetic energy. The expression (6) is rather general, in fact it can be regarded as introduction of the coupling between two systems into the Hamiltonian in the most simple phenomenological way. From this viewpoint the coupling parameter $T_{k}$ is still to be determined through exact solving of the eigenvalue problem for the whole system. Bardeen's approach suggests a simple recipe for calculation of the tunneling parameter $T_{k}$ (also referred as tunneling matrix element) for the case of weak tunneling through potential barrier:

$$
T_{k}=\left\langle\varphi_{k} K \psi\right\rangle-\left\langle\psi K \varphi_{k}\right\rangle^{*},
$$

where $K$ is the kinetic energy operator, commonly equal to

$$
K=-\frac{\hbar^{2}}{2 m} \Delta .
$$

For this problem the 3D integration (7) straightforwardly reduces to integration along any surface inside the barrier. In order to apply the same approach to the problem of the holes tunneling in GaAs it has to be generalized for the case of complex band structure. It would be tempting to do it by treating $K$ in (7) as the kinetic part of the effective Hamiltonian and $\psi, \varphi_{k}$ as envelopes in the basis of Bloch amplitudes. For the valence band of $\operatorname{In}_{x} \mathrm{Ga}_{1-x} \mathrm{As}-$ based heterostructure the holes ground state with total angular momentum $J=3 / 2$ is described by Luttinger Hamiltonian $\left(\hbar k_{x}, \hbar k_{y}, \hbar k_{z}\right.$ are, as usual, the momentum operators along the appropriate axis):

$$
\begin{aligned}
K & =\left(\begin{array}{cccc}
F & H & I & 0 \\
H^{*} & G & 0 & I \\
I^{*} & 0 & G & -H \\
0 & I^{*} & -H^{*} & F
\end{array}\right), \\
F & =-A k^{2}-\frac{B}{2}\left(k^{2}-3 k_{z}^{2}\right), \\
G & =-A k^{2}+\frac{B}{2}\left(k^{2}-3 k_{z}^{2}\right), \\
H & =D k_{z}\left(k_{x}-i k_{y}\right), \\
I & =\frac{\sqrt{3}}{2} B\left(k_{x}^{2}-k_{y}^{2}\right)-i D k_{x} k_{y} .
\end{aligned}
$$


The functions $\psi, \varphi_{k}$ in (7) become 4-component vector functions in the basis (2). Analogously to the simple band case integration over the whole space is reduced to the integration over the surface $\Omega_{S}$ inside the barrier, at that, only $z$-projection of the kinetic energy operator is required. For the eigenfunctions in the QW with zero light hole components the expression for tunneling parameter simplifies into:

$$
T_{k}=(B-A) \int_{\Omega_{S}} d S\left(\varphi_{k}^{*} \frac{d}{d z} \psi-\psi \frac{d}{d z} \varphi_{k}^{*}\right) .
$$

Regrettably, this straightforward generalization of (7) fails to be correct. Indeed, the decay length of wavefunctions (4) is determined by light hole mass while the decay length of the of the QW states (3) is governed by the heavy hole mass. Due to this circumstance the result of the surface integration (10) becomes crucially dependent on the particular position of the integration surface inside the barrier. However, it can be shown that in the case of two masses the exponential dependence of the tunneling parameter $T_{k}$ on the barrier thickness is determined by the smallest mass. The value of $T_{k}$ is also governed by a formfactor corresponding to the overlap of the wavefunctions along the QW plane. We consider the kinetic energy of a hole in the QW being small compared to the QW depth, i.e. $k<<q$. In this case the overlap of the localized wavefunction occur only with the zeroth harmonic of (1). The only nonzero tunneling matrix elements are therefore the following:

$$
\begin{aligned}
& T_{k}=T_{1 k}+T_{2 k} \\
& T_{1 k}=(A-B) \int_{\Omega_{S}} d S\left(R_{0} Y_{00} J_{0}(k \rho) \frac{d}{d z} \eta(z)\right), \\
& T_{2 k}=(A-B) \int_{\Omega_{S}} d S\left(\frac{1}{\sqrt{5}} R_{2} Y_{20} J_{0}(k \rho) \frac{d}{d z} \eta(z)\right),
\end{aligned}
$$

The calculation of these integrals presents no difficulty for any particular surface inside the barrier, however further detalization of these values is beyond the accuracy of the approach. The only statement valid is that the tunneling parameter $T_{k}$ has an exponential dependence on the barrier thickness with the light hole mass entering the exponent index:

$$
T_{k}=(A-B) \zeta(k / q) \frac{\pi^{3 / 4} q \sqrt{k}}{S^{1 / 4}} \exp (-\chi(k / q) q d \sqrt{\beta})
$$

where $1 \leq \chi \leq 2, \zeta \sim 1$ are weak dimensionless functions of $k / q$. Our further analysis will be focused on the case of the hole's kinetic energy being substantially less then the binding energy $E_{0}$, i.e. $k<<q$. For this case it is reasonable to assume that $T_{k}$ does not depend on $k$. However, its rapidly decreasing behavior for $k \gtrsim q$ has to be kept in mind as it provides convergence for any integration over $k$ involving $T_{k}$. For the case $k<<q$ the particular shape of the QW while affecting the particular value of the tunnelimg parameter does not play an important qualitative role and can be assumed rectangular. The interaction with only zeroth cylindrical harmonic means that the continuum spectrum modified by tunneling is non-degenerate. While this fact is not principal for the qualitative results obtained below, it simplifies the mathematics. From the analysis given above we conclude that the tunneling configuration interaction exists only between $\varphi_{k 0,-\frac{3}{2}}$ and $\psi_{-\frac{3}{2}}$, and the same interaction (governed by the parameter $T_{k}$ (11) ) is between $\varphi_{k 0,+\frac{3}{2}}$ and $\psi_{+\frac{3}{2}}$.

\section{THE SPECTRUM MODIFIED BY TUNNELING}

The transfer Hamiltonian (6) with known tunneling parameter (11) allows one to construct the eigenfunctions $\Psi$ of the whole system given those of the localized state $\psi$ and the QW states $\varphi_{k}$ :

$$
\Psi(E)=\nu_{0}(E) \psi+\sum_{k} \nu_{k}(E) \varphi_{k}
$$

$E$ denotes the energy of the state $\Psi$. Here $\varphi_{k}$ are the wavefunctions with zeroth cylindrical harmonic, as was shown above the other harmonics are not affected by the tunneling configuration interaction. Plugging (12) into the stationary Schrodinger equation:

$$
H \Psi=E \Psi
$$

with $H$ being the effective Hamiltonian (6) one gets the following system of linear equations:

$$
\begin{aligned}
& \nu_{0} \varepsilon_{0}+\sum_{k} \nu_{k} T_{k}^{*}=E \nu_{0} \\
& \nu_{k} \varepsilon_{k}+T_{k} \nu_{0}=E \nu_{k}
\end{aligned}
$$


Solving the eignenvalue problem for system (13) one can get the spectrum and the coefficients $\nu_{0}, \nu_{k}$, i.e. the eigenfunctions of the system. Transition from discrete set of states $\nu_{k}(E)$ to continuous function $\nu(\varepsilon, E)$ is straightforward (as the continuum states are non-degenerate we can use the energy $\varepsilon$ instead of $k$ as the quantum number of the state). Instead of (12) and (13) we write:

$$
\begin{aligned}
& \Psi(E)=\nu_{0}(E) \psi+\int_{0}^{\infty} \nu(E, \varepsilon) \varphi(\varepsilon) d \varepsilon \\
& \nu_{0}(E) \varepsilon_{0}+\int_{0}^{\infty} t(\varepsilon) \nu(E, \varepsilon) d \varepsilon=E \nu_{0}(E), \\
& \nu(E, \varepsilon) \varepsilon+t(\varepsilon) \nu_{0}(E)=E \nu(E, \varepsilon) .
\end{aligned}
$$

The normalizations for $\psi$ and $\varphi(\varepsilon)$ are:

$$
\begin{aligned}
& \left\langle\psi\left(\varepsilon_{0}\right) \mid \psi\left(\varepsilon_{0}\right)\right\rangle=1, \\
& \left\langle\varphi(\varepsilon) \mid \varphi\left(\varepsilon^{\prime}\right)\right\rangle=\delta\left(\varepsilon-\varepsilon^{\prime}\right) .
\end{aligned}
$$

With the chosen normalization the discrete tunneling parameter $T_{k}$ and the one entering (15) are related as follows:

$$
T_{k}^{2} N_{0}(\varepsilon)=t^{2}(\varepsilon),
$$

where

$$
N_{0}(\varepsilon)=\sqrt{\frac{m_{h h^{\prime} S}^{\prime}}{2 \pi^{3} \varepsilon \hbar^{2}}}
$$

is the density of states for the selected basis of states with zeroth cylindrical harmonic. The discrete system (13) is an eigenvalue problem, but the continuous problem (15) is not. In the present work we consider the case of the localized energy level lying within the range of the continuum: $\varepsilon_{0}>>t^{2}$. For this case the solution is obtained as shown in $\stackrel{1}{*}$ :

$$
\begin{aligned}
\nu_{0}^{2}(E) & =\frac{t^{2}(E)}{\pi^{2} t^{4}(E)+\left(E-\widetilde{\varepsilon_{0}}\right)^{2}}, \\
\nu(E, \varepsilon) & =\nu_{0}(E)\left(P \frac{t(\varepsilon)}{E-\varepsilon}+Z(E) t(E) \delta(E-\varepsilon)\right),
\end{aligned}
$$

where

$$
\begin{aligned}
& Z(E)=\frac{E-\varepsilon_{0}-F(E)}{t^{2}(E)}, \\
& F(E)=\int_{0}^{\infty} P \frac{t^{2}(\varepsilon)}{(E-\varepsilon)} d \varepsilon
\end{aligned}
$$

$P$ stands for the principal value and $\tilde{\varepsilon}_{0}$ is the center of configuration resonance, which appears to be slightly shifted from $\varepsilon_{0}$ :

$$
\tilde{\varepsilon}_{0}(E)=\varepsilon_{0}+F(E) .
$$

Because of $k<<q$ it is reasonable to treat $t=$ const everywhere, except for (20) where decrease of $t$ at $E \rightarrow \infty$ is necessary for convergence of the integral. In order to analyze the influence of the configuration interaction on the luminescence spectra we have to calculate matrix element of operator $\hat{M}$ describing interband radiative transitions between the hybridized hole wavefunction $\Psi(E)$ and wavefunction of an electron in the quantum well of the conductance band $\xi_{k_{e} l_{e}}$, here $k_{e}$ is the magnitude of the electron wavevector, $l_{e}$ is the number of cylindrical harmonic analogously to (11). We assume that (a) there are no radiative transitions between the localized hole wavefunction $\psi$ and the 2D electron wavefunction $\xi_{k_{e} l_{e}}$ thus the matrix element for transitions from the localized state:

$$
\left\langle\xi_{k_{e} l_{e}}|\hat{M}| \psi\right\rangle=0
$$

(b) the interband radiative transitions between the free 2D states in the QW are direct, the matrix element given by:

$$
M_{0}=\left\langle\xi_{k_{e} l_{e}}|\hat{M}| \varphi_{k l}\right\rangle=u_{k} \delta\left(k-k_{e}\right) \delta_{l, l_{e}}
$$


where $u_{k}$ is the appropriate dipole matrix element. With use of (14), (19), (22) and (23) we arrive to the the matrix element for transitions between states $\Psi(E)$ and $\xi_{k_{e} 0}$ (according to previous notes this matrix element differs from $M_{0}$ only for the zeroth cylindrical harmonic) :

$$
M=\left\langle\xi_{k_{e} 0}|\hat{M}| \Psi(E)\right\rangle=\nu\left(E, \alpha \varepsilon_{e}\right) u\left(\alpha \varepsilon_{e}\right),
$$

$\alpha=m_{e} / m_{h h}^{\prime}$, where $m_{e}$ is the effective in-plane electron mass, $\varepsilon_{e}=\hbar^{2} k_{e}^{2} / 2 m_{e}$. The particular form of M (24) prevents from calculation of the ratio $M^{2} / M_{0}^{2}$ as done in the classical Fano resonance calculations $\mathrm{I}^{\underline{\underline{1}} \text {. The latter }}$ assumes unperturbed matrix element $M_{0}$ to be constant which is obviously not the case for the direct transitions demanding the momentum conservation (23). In our case the ratio $M^{2} / M_{0}^{2}$ doesn't readily give physically meaningful result due to the delta function in (19), one rather have to proceed to calculation of an observable. With the Fermi's Golden Rule for the transition probability we write:

$$
W(\hbar \omega)=\frac{2 \pi}{\hbar} \int_{0}^{\infty} \int_{0}^{\infty}\left|M\left(E^{\prime}, \varepsilon_{e}\right)\right|^{2} f_{e}\left(\varepsilon_{e}\right) f_{h}\left(E^{\prime}\right) \delta\left(E^{\prime}+\varepsilon_{e}+E_{g}-\hbar \omega\right) d E^{\prime} d \varepsilon_{e},
$$

where $E_{g}$ is the bandgap and $\hbar \omega$ - energy of radiated photon, $f_{e}, f_{h}$ - the energy distribution functions for the electrons and holes respectively. To deal properly with the delta function entering $M^{2}$ in (24) and emerging in (25) we pass on to averaging the $W(\hbar \omega)$ over a small spectral interval of width $\Omega$ centered at $\omega_{0}$ :

$$
\widetilde{W}\left(\hbar \omega_{0}\right)=\frac{1}{\Omega} \int_{\omega_{0}-\Omega / 2}^{\omega_{0}+\Omega / 2} W(\hbar \omega) d \omega .
$$

Using (24) and (19) we obtain:

$$
\widetilde{W}\left(\hbar \omega_{0}\right)=\frac{2 \pi}{\hbar} \frac{1}{\hbar \Omega} \int_{\frac{\hbar \omega_{0}-E_{g}-\hbar \Omega / 2}{1+\alpha^{-1}}}^{\frac{\hbar \omega_{0}-E_{g}+\hbar \Omega / 2}{1+\alpha-1}}\left[N\left(E^{\prime}\right)-\frac{1}{t^{2}\left(E^{\prime}\right)\left(\pi^{2}+Z^{2}\left(E^{\prime}\right)\right)}\right] u^{2}\left(\alpha^{-1} E^{\prime}\right) f\left(E^{\prime}\right) d E^{\prime},
$$

where $f\left(E^{\prime}\right)=f_{e}\left(\alpha^{-1} E^{\prime}\right) f_{h}\left(E^{\prime}\right)$. The first term in brackets describes the transition rate for radiative recombination in the QW with no account for the tunneling, therefore $N\left(E^{\prime}\right)$ here is the total density of states (including not only the zeroth but all cylindrical harmonics):

$$
N\left(E^{\prime}\right)=\frac{m_{h h^{\prime}}^{\prime} S}{2 \pi \hbar^{2}}
$$

Integration assuming the functions $\widetilde{\varepsilon}_{0}, t, u, f$ being constant within the range of integration $(t(E) \equiv t, u(E) \equiv u$ are assumed constant everywhere) yields :

$$
\widetilde{W}\left(\hbar \omega_{0}\right)=\frac{2 \pi}{\hbar} u^{2} f(E)\left[\frac{m_{h h}^{\prime} S}{2 \pi \hbar^{2}}-\frac{1}{\hbar \Omega \pi}\left[\arctan \frac{\Delta E+w}{\pi t^{2}}-\arctan \frac{\Delta E-w}{\pi t^{2}}\right]\right],
$$

where

$$
\begin{aligned}
& E=\frac{\hbar \omega_{0}-E_{g}}{1+\alpha^{-1}} \\
& \Delta E=E-\widetilde{\varepsilon_{0}}(E) \\
& w=\frac{\hbar \Omega}{2\left(1+\alpha^{-1}\right)}
\end{aligned}
$$

We assume weak tunneling, $t^{2}$ being the smallest energy scale. In the vicinity of resonance

$$
\Delta E \in\left(-w+t^{2}, w-t^{2}\right),
$$

expansion of (28) to the first order in $t^{2}$ gives:

$$
\widetilde{W}\left(\hbar \omega_{0}\right)=\frac{2 \pi}{\hbar} u^{2} f(E)\left[\frac{m_{h h}^{\prime} S}{2 \pi \hbar^{2}}-\frac{1}{\hbar \Omega}+\frac{1}{1+\alpha^{-1}} \frac{t^{2}}{w^{2}-(\Delta E)^{2}}\right] .
$$




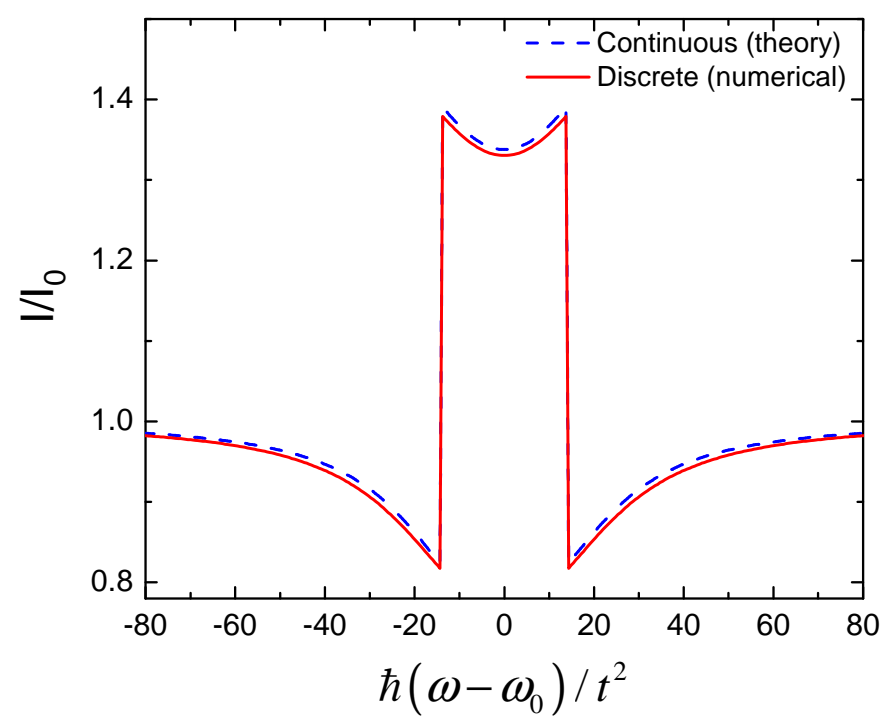

FIG. 1: (Color online) Modification of the luminescence spectrum by tunneling configuration interaction: numerical calculation for the discrete levels (solid line) and analytical formula (32) (dashed line)

Note that (31) has a term $-1 / \hbar \Omega$ which does not depend on the tunneling. Its appearance is due to peculiarity of the mathematics of the Fano model reflected in (19). When a non-interacting state with energy $\varepsilon_{0}$ is appended to the system so that $\varepsilon_{0}$ lies within its spectrum, one of the energy levels of the whole system becomes doubly degenerate. This fact is not properly accounted for in (19) and one state is lost. It should be added back manually to the spectral density by canceling the second term in (31). Treating the same issue in a different way, one should examine $\Delta \widetilde{W}=\widetilde{W}-\widetilde{W}_{0}$ instead of $W$ itself, $\widetilde{W}_{0}$ being the unperturbed transition rate: (28) evaluated for $t=0$. In a similar way studying the ratio of matrix elements in original Fano work ${ }^{1}$ circumvents the disappearance of one level.

The results obtained for a single impurity can be also applied to an ensemble of impurities provided their interaction between each other is weak compared to the tunnel coupling with the QW. If the concentration of the impurities is low enough to produce only weak perturbation of the luminescence spectra, we can simply multiply the tunneling term by the number of impurities. After normalization by the area of the QW we finally get the spectral density of the luminescence intensity:

$$
I\left(\hbar \omega_{0}\right)=\frac{2 \pi}{\hbar} u^{2} f(E)\left[\frac{m_{h h}^{\prime}}{2 \pi \hbar^{2}}+\frac{n}{\pi \hbar \Omega}\left[\arctan \frac{\Delta E-w}{\pi t^{2}}-\arctan \frac{\Delta E+w}{\pi t^{2}}-\pi \frac{\operatorname{sgn}(\Delta E-w)-\operatorname{sgn}(\Delta E+w)}{2}\right]\right],
$$

where $n$ is the 2D concentration of impurities. The last term in brackets corrects the lost level issue to provide exact canceling of the perturbation of the spectra at $t=0$. For high concentration of the impurities the formula (32) may give a meaningless result (the intensity may become negative at some points). Indeed for high concentration the real physical picture becomes slightly different - interaction between the impurities splits their energy levels forming a small range of discrete levels, accordingly, the configuration resonances become slightly shifted. Taking this effect into account eliminates the puzzling behavior of (32) at high concentration but does not affect the answer for the calculation of polarization given in the next section.

The analytical result (32) was verified by numerical simulation performed for the discrete system (13). The system was solved for 500 discrete levels with interlevel separation $10^{-5} \mathrm{eV}$, the discrete tunneling parameter was taken $T_{k}=3.3 \cdot 10^{-5} \mathrm{eV}$, which corresponds to the continuous tunneling parameter $t^{2}=10^{-4} \mathrm{eV}$, the other relevant parameters were: $w=5 \cdot 10^{-4} \mathrm{eV}, n=10^{10} \mathrm{~cm}^{-2}, m_{e}=0.03 m_{0}, m_{h h}=0.5 m_{0}, m_{h h}^{\prime}=0.15 m_{0}$. In both calculations all the states were assumed fully occupied, i.e. the energy distribution function was kept $f(E)=1$. Analogously to (23) the matrix element for the discrete system was taken: $M_{k}\left(\varepsilon, \varepsilon_{e}\right)=u_{k} \delta_{k, k_{e}} \delta_{l, l_{e}}$. The calculation result presented in Fig 1 demonstrates perfect agreement with the analytical expression (32) and confirms the validity of the latter. The considered tunneling configuration interaction thus gives rise to the luminescence intensity within a certain spectral range (30) corresponding to the resonance. This increase is compensated by the decrease of the intensity outside of the this range as can be seen in Fig 1 The width of the resonance is determined by $\Omega$ which has the meaning of spectral resolution of the measurement setup. However, for comparison with experimental spectra the inhomogeneous broadening should be accounted for as it usually exceeds the instrumental spectral resolution. An 
expression for the integral intensity over the whole spectra reads:

$$
I=\frac{2 \pi}{\hbar} \int_{0}^{\infty} u^{2} f(E)\left[\frac{m_{h h}^{\prime}}{2 \pi \hbar^{2}}-\frac{n t^{2}(E)}{\pi^{2} t^{4}(E)+\left(E-\widetilde{\varepsilon_{0}}\right)^{2}}+n \delta\left(E-\widetilde{\varepsilon_{0}}\right)\right] d E .
$$

This formula follows from (25) in the same way as (28) and (31) were obtained. The delta-function here is added manually to treat the lost level issue - it provides canceling of the second term in the limit $t \rightarrow 0$ and thus gives the correct expression in the absence of tunneling:

$$
I_{0}=\frac{2 \pi}{\hbar} \int_{0}^{\infty} u^{2} f(E) \frac{m_{h h}^{\prime}}{2 \pi \hbar^{2}} d E .
$$

Note that the spectral width of the resonance $\Omega$ does not enter the expression for integral intensity (33).

\section{POLARIZATION OF THE SPECTRA}

While the redistribution of the spectral density does not change the integral intensity it gives rise to the integral polarization of the spectra in the magnetic field as illustrated by Fig 2 The 2D holes with projection of total angular momentum $j=+3 / 2$ and $j=-3 / 2$ recombine emitting respectively right- $\left(\sigma^{+}\right)$and left- $\left(\sigma^{-}\right)$circularly polarized light. In section III it was shown that the heavy holes with $j=-3 / 2$ interact basically with the eigenfunction $\psi_{-\frac{3}{2}}$ of the localized state. Let us denote the corresponding energy of this state $\varepsilon_{0}^{-}$. The $2 \mathrm{D}$ holes with $j=+3 / 2$ interact in turn with $\psi_{+\frac{3}{2}}$ which corresponds to the energy $\varepsilon_{0}^{+}$. An external magnetic field applied in $z$ would cause Zeeman splitting between $\varepsilon_{0}^{+}$and $\varepsilon_{0}^{-}$. The splitting $\Delta=\varepsilon_{0}^{+}-\varepsilon_{0}^{-}$may also originate from exchange interaction of holes with spin-polarized Mn ions. The value of $\Delta$ in this case is determined by exchange constant and depend on the degree of Mn spin polarization. The splitting of the localized energy level leads, in turn, to the splitting of the configuration resonance. Indeed, as follows from (26), (28), (20) the difference in the positions of the resonances $E_{+}$ and $E^{-}$corresponding to the localized levels $\varepsilon_{0}^{+}$and $\varepsilon_{0}^{-}$is given by:

$$
\widetilde{\Delta}=E^{+}-E^{-}=\Delta+t^{2} \ln \left(1+\frac{\widetilde{\Delta}}{E_{-}}\right) .
$$

Unless the position of the resonance $E^{-}$is too close to the valence band edge the last term in (35) can be neglected and $\widetilde{\Delta}=\Delta=\varepsilon_{0}^{+}-\varepsilon_{0}^{-}$. The applicability of this result is limited to the case $\varepsilon_{0}>\Delta$. This condition, in fact, simply means that the splitting of the localized level does not bring any of the sublevels beyond the energy range of the $2 \mathrm{D}$ continuum so that the exploited Fano approach remains valid. Our consideration will be always limited to this case. With account of the energy distribution functions for the holes and electrons the shifted positions of the resonances lead to the difference in the luminescence intensity for the opposite circular polarizations. Let $I^{+}, I^{-}$be the integral luminescence intensities of circular polarizations $\sigma^{+}$and $\sigma^{-}$respectively. Assuming $\left|I^{ \pm}-I_{0}\right|<<I_{0}$ the integral polarization is given by:

$$
P=\frac{I^{+}-I^{-}}{2 I_{0}}
$$

With use of (33) this yields:

$$
P=\frac{n \pi \hbar^{2}}{m_{h h^{\prime}}} \frac{f\left(\widetilde{\varepsilon}_{0}^{-}\right)-f\left(\widetilde{\varepsilon}_{0}^{+}\right)+\int_{0}^{\infty}\left[\frac{t^{2}(E) f(E)}{\pi^{2} t^{4}(E)+\left(E-\widetilde{\varepsilon}_{0}^{+}\right)^{2}}-\frac{t^{2}(E) f(E)}{\pi^{2} t^{4}(E)+\left(E-\widetilde{\varepsilon}_{0}^{-}\right)^{2}}\right] d E}{\int_{0}^{\infty} f(E) d E} .
$$

The slow varying functions in the upper integrals may be assumed as constants taken at $\widetilde{\varepsilon}_{0}^{-}$, $\widetilde{\varepsilon}_{0}^{+}$, the tunneling parameter will be treated as a constant in the whole range of interest $t^{2}(E) \equiv t^{2}$.

Then expanding over $t^{2}$ gives for the first order term:

$$
P=\frac{n \pi \hbar^{2} t^{2}}{m_{h h}^{\prime}} \frac{f\left(\widetilde{\varepsilon}_{0}^{-}\right)\left(\widetilde{\varepsilon}_{0}^{-}\right)^{-1}-f\left(\widetilde{\varepsilon}_{0}^{+}\right)\left(\widetilde{\varepsilon}_{0}^{+}\right)^{-1}}{\int_{0}^{\infty} f(E) d E}
$$




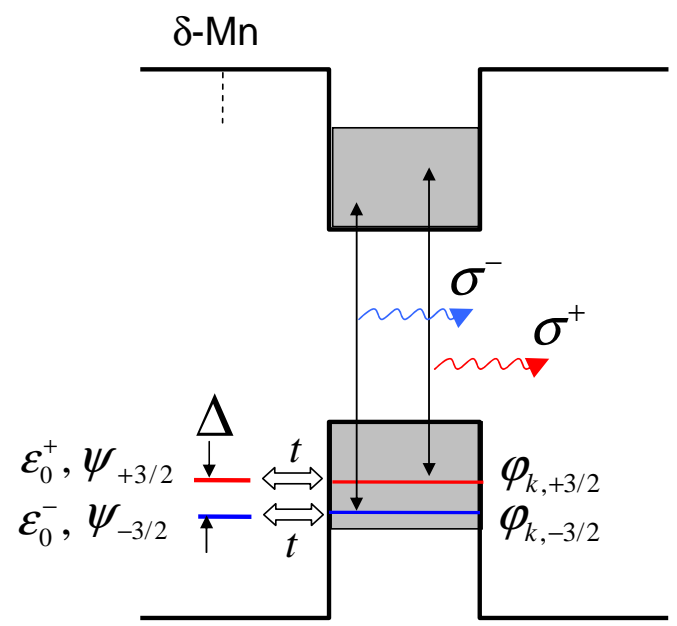

FIG. 2: (Color online) Mechanism of polarization of the luminescence. The localized hole levels split in magnetic field. Each of them effectively couples with the $2 \mathrm{D}$ holes having certain projection of angular momentum. Shifted positions of the resonances with account for temperature distribution of the holes cause the difference in intensities of circular polarizations $\sigma^{+}, \sigma^{-}$

The formula (37) leaves not much room for further simplification for a general case of $f_{e}(E), f_{h}(E)$ being two Fermi distributions characterized by chemical potentials $\mu_{e}, \mu_{h}$ and the temperatures $T_{e}$ and $T_{h}$ respectively, all four parameters being different. Let us analyze a few particular cases leading to compact analytical expressions for P. All the cases imply $\widetilde{\varepsilon_{0}}>\Delta$. Firstly, let the holes be fully degenerate and both energies $\widetilde{\varepsilon}_{0}^{-}$and $\widetilde{\varepsilon}_{0}^{+}$lying well beyond the quasi Fermi level of the holes so that: $\mu_{h}-\widetilde{\varepsilon}_{0}^{+}>>$. In this case the distribution function of the holes can be considered as constant $F_{h}(E)=1$ in the range $E \in\left(\widetilde{\varepsilon}_{0}^{-}, \widetilde{\varepsilon}_{0}^{+}\right)$Assuming the electrons to be non-degenerate with their temperature $T_{e}$ the formula (37) reduces to:

$$
P=P_{1} e^{-\frac{\widetilde{\sigma_{0}}}{k T^{*}}} \sinh \frac{\Delta}{2 k T^{*}},
$$

where

$$
P_{1}=\frac{2 \pi n \hbar^{2} t^{2}}{m_{h h}^{\prime} \widetilde{\varepsilon_{0}} k T^{*}}
$$

Here $T^{*}=\alpha T_{e}$. Exactly the same expression is valid for the case when both electrons and holes are non-degenerate. The only difference from the previously considered case is that now the effective temperature $T^{*}$ is given by

$$
\frac{1}{T^{*}}=\left(\frac{1}{\alpha T_{e}}+\frac{1}{T}\right) .
$$

The expression (38) is plotted in Fig $\mathbb{V}$ for different values of the parameter $\gamma \equiv \Delta / \widetilde{\varepsilon_{0}}$. The polarization shows nonmonotonous behavior with increasing the temperature. In the discussed theory the polarization arises from splitting of the configuration resonances positions for $\sigma^{+}$and $\sigma^{-}$spectra. The configuration resonance itself causes the redistribution of the transitions rate in the vicinity of the resonance energy conserving the total rate, thus the net polarization is subect to the difference in occupation numbers for $\widetilde{\varepsilon}_{0}^{-}$and $\widetilde{\varepsilon}_{0}{ }^{+}$. The maximum integral polarization is therefore naturally expected when the derivative of the combined distribution function $f(E)$ reaches its maximum value within the range $E \in\left(\widetilde{\varepsilon}_{0}^{-}, \widetilde{\varepsilon}_{0}^{+}\right)$. For the considered case the maximum of the derivative is at $\widetilde{\varepsilon}_{0}$ when $\widetilde{\varepsilon}_{0}=k T^{*}$ and the value of the derivative decreases with increase of $\widetilde{\varepsilon}_{0}$. This explains the overall decrease of the maximum polarization with decrease of $\gamma$ in Fig $\left[\mathbf{V}\right.$ For another case we consider the electrons distribution function $f_{e}$ being nearly constant within the configuration resonances. This can be due to their non-equilibrium distribution with a high quasi Fermi level or the electrons temperature $T_{e}$ being much higher than that of the holes. The holes are now considered to have Fermi distribution function with the quasu Fermi level $\mu_{h}$ and the temperature $T$. We also assume $k T<<\widetilde{\varepsilon}_{0}$. In this case from (37) we get:

$$
P=P_{0}\left(\frac{2 \exp (\beta \xi) \sinh (\xi / 2)+\gamma}{\exp (2 \beta \xi)+2 \exp (\beta \xi) \cosh (\xi / 2)+1}\right),
$$




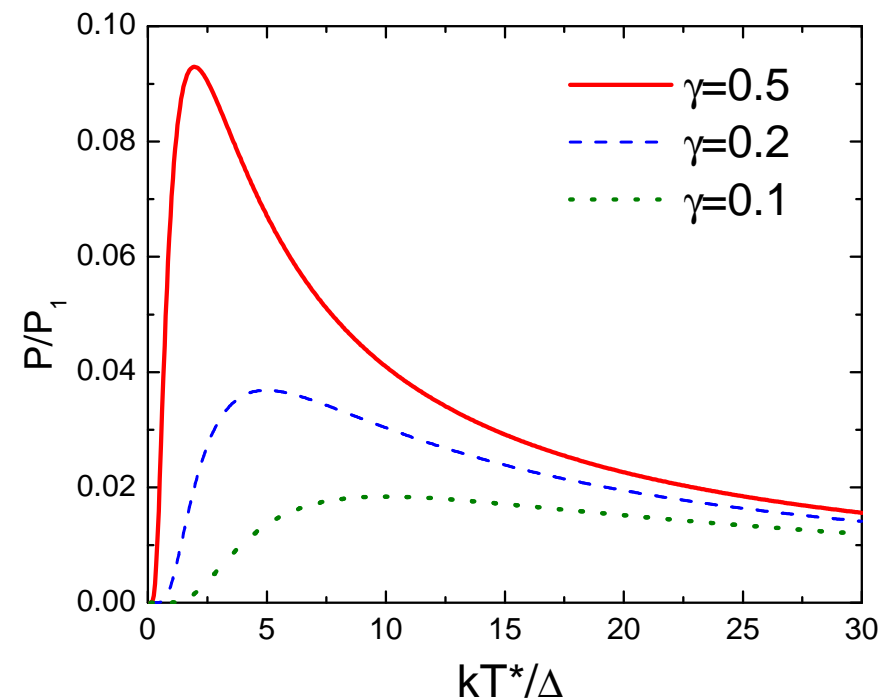

FIG. 3: (Color online) Temperature dependence of integral polarization. Electrons are non-degenerate, holes are either nondegenerate or have the con

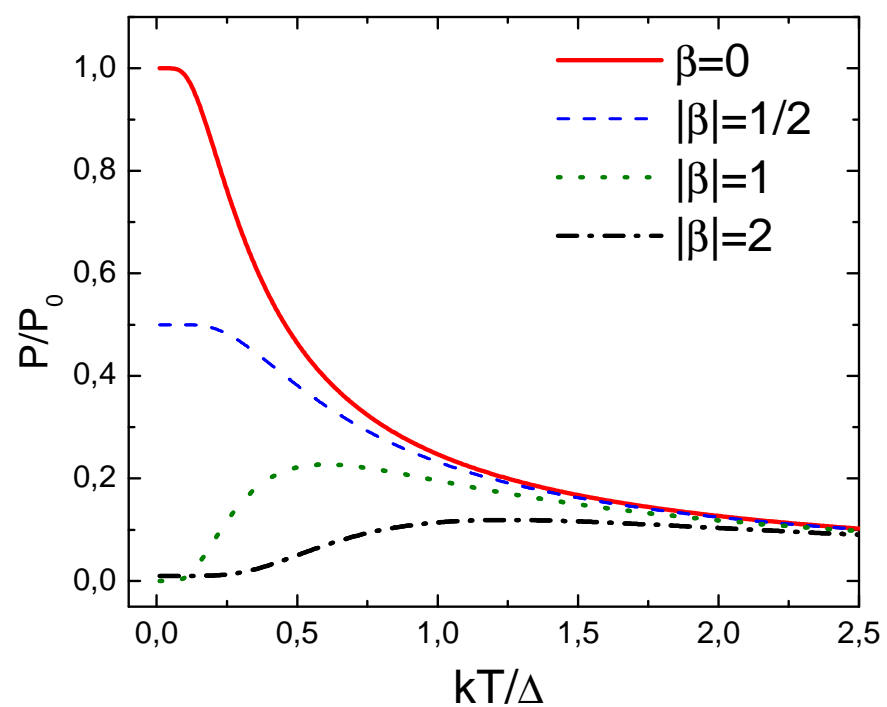

FIG. 4: (Color online) Temperature dependence of polarization for the case of electrons distribution function being constant within the configuration resonances. The parameter $\beta \equiv \frac{\widetilde{\varepsilon_{0}}-\mu_{h}}{\Delta}$ denotes deviation of the holes Fermi level from the configuration resonance, $\gamma=0.1$.

where

$$
\begin{aligned}
& \beta=\frac{\widetilde{\varepsilon_{0}}-\mu_{h}}{\Delta}, \\
& \xi=\Delta / k T, \\
& P_{0}=\frac{n \pi \hbar^{2} t^{2}}{m_{h h}^{\prime} \mu_{h}^{2}} .
\end{aligned}
$$

The dependence (39) of $P / P_{0}$ on $1 / \xi$ is plotted in Fig $\mathbb{V}$ for different values of the parameter $\beta$ (the value of $\gamma$ was taken 0.1 . In this case the maximum of the distribution function derivative is at the holes Fermi level $\mu_{h}$, therefore the largest integral polarization corresponds to $\beta=0$. For this particular case (39) simplifies into: 


$$
P=P_{0}\left(\tanh (\xi)+\frac{\gamma}{2 \cosh ^{2}(\xi)}\right)
$$

The integral polarization obtained within given approach can be alternatively expressed through an effective g-factor of the holes $g_{\text {eff }}$. Let us consider Zeeman term in the Hamiltonian of the 2D holes:

$$
H_{B}=\mu_{0} g_{e f f} J_{z} B
$$

where $J_{z}$ is the angular momentum projection operator, $\mu_{0}$ is the Bohr magneton and B is the magnetic field applied along $z$-direction. The polarization of $2 \mathrm{D}$ holes due to the splitting between the two subbands with $j=+3 / 2$ and $j=-3 / 2$ is given by:

$$
P_{B}=\frac{\int_{0}^{\infty}\left(f\left(E-\Delta_{B} / 2\right)-f\left(E+\Delta_{B} / 2\right)\right) d E}{\int_{0}^{\infty}\left(f\left(E-\Delta_{B} / 2\right)+f\left(E+\Delta_{B} / 2\right)\right) d E}
$$

where $\Delta_{B}=3 \mu_{0} g_{\text {eff }} B$. For the nondegenerate case one gets

$$
P_{B}=\tanh \frac{\Delta_{B}}{2 k T^{*}}
$$

Comparing (43) with (38) gives:

$$
g_{e f f}=\frac{2 k T^{*}}{3 \mu_{0} B} \tanh ^{-1}\left[\left(\frac{2 \pi n \hbar^{2} t^{2}}{m_{h h^{\prime}} \widetilde{\varepsilon_{0}} k T^{*}}\right) e^{-\frac{\widetilde{\varepsilon_{0}}}{k T^{*}}} \sinh \frac{\Delta_{0}}{2 k T^{*}}\right]
$$

In the same way an expression for the degenerate case can be easily obtained.

\section{DISCUSSION}

The key advantage of the Fano approach utilized in the present study is that the unknown eigenfunctions of the complex system are expressed through the known ones of the uncoupled states, in our case these are the hole localized at $\mathrm{Mn}$ and the free 2D hole in the QW. Given the expansion (12) any effects on the localized state can be easily translated into effect for the whole coupled system. For the polarization effect under study the key parameters are the energy of the localized level $\varepsilon_{0}$, the splitting parameter $\Delta$ and the tunneling parameter $t^{2}$. The binding energy for a hole at a single $\mathrm{Mn}$ in GaAs is known to be $E_{0} \approx 110 \mathrm{meV} 14$. For the enhanced Mn concentrations in the delta-layer up to $10^{13} \mathrm{~cm}^{-2}$ the impurity band is established with the binding energy lowering down to $50 \mathrm{meV}$ or even less ${ }^{15}-17$. Thus we consider the QW depth for the holes starting from $50 \mathrm{meV}$ to be likely for the resonance tunneling effects under study to occur. Estimations for the splitting energy $\Delta$ subject to both exchange interaction between the hole and $\mathrm{Mn}$ and external magnetic field. For small concentration of Mn the splitting between the states having opposite projections of the angular momentum can be simply estimated as $\Delta=\mu_{0} g B$ with $B$ being an external magnetic field and $g \approx 3$ is the g-factor for the hole at Mn. This makes $\Delta \sim 0.1 \mathrm{meV}$ for $B \sim 1 \mathrm{~T}$. Samples with higher Mn concentrations up to a few percent are known to exhibit ferromagnetic properties 16 , in this case the levels splitting $\Delta$ is to be considered with account for $\mathrm{p}$-d exchange interaction 16 . The particular value of $\Delta$ for typical experimental samples still remains questionable, in the ferromagnetic regime the splitting is probably believed to be in the range $\Delta \sim 1-10 \mathrm{meV}$ by the order of magnitude. The magnitude of the tunnel coupling is, of course, the key parameter determining the polarization. From (11) and (18) it follows that the tunneling parameter can be estimated as

$$
t^{2}=(A-B)^{2} \frac{2 m_{h h} m_{h h}^{\prime} E_{0}}{\hbar^{4}} e^{-\frac{2 \sqrt{2 m_{l h} E_{0}}}{\hbar} d} .
$$

Substituting band parameters for GaAs $B-A \approx 1 \cdot \hbar^{2} / 2 m_{0}, E_{0}=100 \mathrm{meV}, d=4$ nm one obtains the characteristic value $t^{2} \sim 0.1 \mathrm{meV}$. We then take the $\mathrm{Mn}$ concentration $n \sim 10^{12} \mathrm{~cm}^{-2}, \xi=1, \varepsilon_{0} \sim 3 \mathrm{meV}, \beta=0$. This set of parameters gives $P_{0} \approx 0.2, P_{1} \approx 0.6$. The experimental temperature dependence of polarization obtained in 10 qualitatively agrees with (41). Beside the analytical expressions for the general case (37) and particular cases (38), (41), a numerical simulation of the luminescence spectra can be performed based on (32). An example of such calculation is shown in Fig.VI. For the calculation the following parameters were taken: $\Delta=1 \mathrm{meV}, n=10^{11} \mathrm{~cm}^{-2}$, $T=T_{e}=20 \mathrm{~K}, \varepsilon_{0}=\mu_{h}=1 \mathrm{meV}$, the inhomogeneous broadening of the spectra was accounted for by normal 


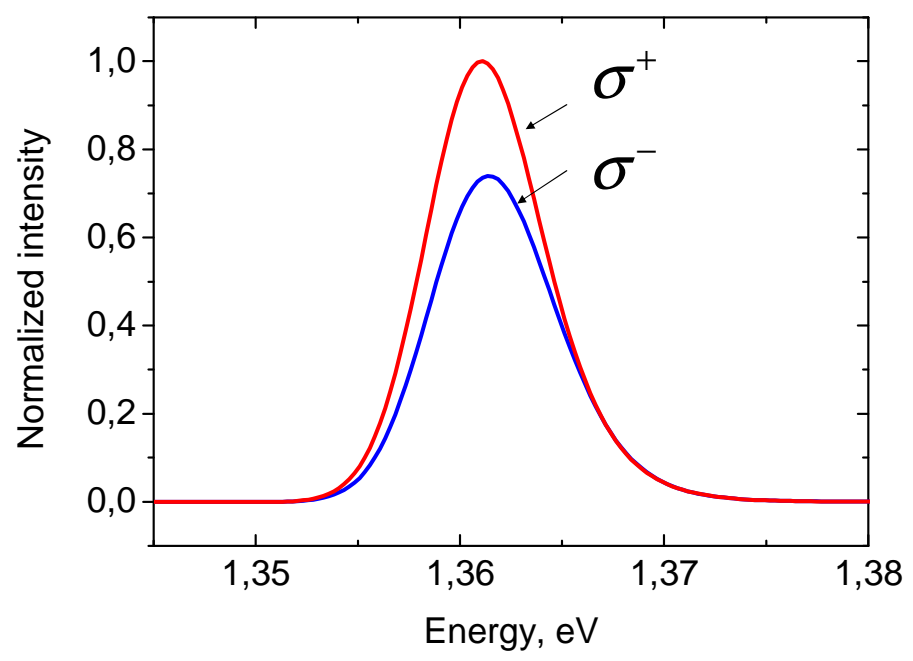

FIG. 5: (Color online) An example of calculated luminescence spectra for right $\left(\sigma^{+}\right)$and left $\left(\sigma^{-}\right)$circular polarizations. The parameters used in calculations are given in the text.

distribution of $E_{g}$ with dispersion taken as $\sigma=3 \mathrm{meV}$ (corresponds to the fluctuation of the QW width by half a monolayer). The calculated spectra presented in Fig VI seem to be in good agreement with the experimental results obtained in ${ }^{9} 10$.

As mentioned in section [1] the magnetic field in the developed theory was assumed nonquantizing. Indeed an estimate for the energy of Landau levels separation gives:

$$
\hbar \omega_{c}=\frac{e \hbar B}{m_{h h}^{\prime} c} \approx 0.3 \mathrm{meV}
$$

for the magnetic field $B=0.5 \mathrm{~T}$. This value is substantially less than the typical kinetic energy of the holes estimated as $\varepsilon \approx 1-10 \mathrm{meV}$. However, this value is comparable with the tunneling parameter $t^{2}$. Therefore for the experimental data the validity of the developed theory is well justified for $B \lesssim 0.5 \mathrm{~T}$.

\section{SUMMARY}

We have presented a theory treating tunneling configuration interaction between a continuum of states in the QW and a paramagnetic impurity located outside of the QW. The coupling between the localized state and the QW is described by means of tunneling Hamiltonian. We utilized the well known Fano approach for calculation of the matrix elements for direct radiative transitions between electrons and holes in the QW. At that the new results were obtained not covered by the conventional Fano formula 1.2 , the tunneling interaction between the localized hole state and continuum of states in the QW results in the symmetrical redistribution of the luminescence spectral density in the vicinity of the resonance. While this redistribution does not affect integral luminescence intensity, it causes an integral circular polarization of the light emitted from the QW provided the localized state is split in the projection of the hole angular momentum under external magnetic field or due to exchange interaction with $\mathrm{Mn}$ ions. The presented theory expresses the eigen states of the system with weak tunnel coupling through the wavefunctions of the hole localized at paramagnetic center and the 2D states of the continuum. For this reason it seems to be capable of describing other effects expected in such systems like an anisotropy of the holes g-factor in the QW induced by a paramagnetic impurity or the indirect exchange interaction between the localized hole states provided by the continuum located at a tunneling distance.

\section{ACKNOWLEDGEMENTS}

We thank V. D. Kulakovskii for very fruitful discussions and also express our thanks to B. A. Aronzon, P. I. Arseev, V. L. Korenev, V. F. Sapega for useful and helpful comments. The work has been supported by RFBR (grants no 09- 
02-00469, 11-02-00348, 11-02-00146, 12-02-00815,12-02-00141), Russian Ministry of Education and Science (contract N 14.740.11.0892).

* Electronic address: rozhansky@gmail.com

1 U. Fano, Phys. Rev. 124, 1866 (1961).

2 A. E. Miroshnichenko, S. Flach, and Y. S. Kivshar, Rev. Mod. Phys. 82, 2257 (2010).

3 A. Blom, M. A. Odnoblyudov, I. N. Yassievich, and K. A. Chao, Phys. Rev. B 65, 155302 (2002).

${ }^{4}$ V. I. Okulov, A. T. Lonchakov, T. E. Govorkova, K. A. Okulova, S. M. Podgornykh, L. D. Paranchich, and S. Y. Paranchich, Low Temperature Physics 37, 220 (2011), URL http://link.aip.org/link/?LTP/37/220/1.

5 V. Aleshkin, L. Gavrilenko, M. Odnoblyudov, and I. Yassievich, Semiconductors 42, 880 (2008), ISSN 1063-7826, 10.1134/S1063782608080034, URL http://dx.doi.org/10.1134/S1063782608080034.

${ }^{6}$ V. Korenev, JETP Letters $\mathbf{7 8}, \quad 564 \quad$ (2003), $\quad$ ISSN $0021-3640, \quad 10.1134 / 1.1641485, \quad$ URL http://dx.doi.org/10.1134/1.1641485

7 B. A. Aronzon, M. V. Kovalchuk, E. M. Pashaev, M. A. Chuev, V. V. Kvardakov, I. A. Subbotin, V. V. Rylkov, M. A. Pankov, I. A. Likhachev, B. N. Zvonkov, et al., Journal of Physics: Condensed Matter 20, 145207 (2008), URL http://stacks.iop.org/0953-8984/20/i=14/a=145207

8 B. A. Aronzon, M. A. Pankov, V. V. Rylkov, E. Z. Meilikhov, A. S. Lagutin, E. M. Pashaev, M. A. Chuev, V. V. Kvardakov, I. A. Likhachev, O. V. Vihrova, et al., 107, 023905 (2010), ISSN 00218979, URL http://dx.doi.org/10.1063/1.3267314

9 M. Dorokhin, S. Zaitsev, A. Brichkin, O. Vikhrova, Y. Danilov, B. Zvonkov, V. Kulakovskii, M. Prokofeva, and A. Sholina, Physics of the Solid State 52, 2291 (2010), ISSN 1063-7834, 10.1134/S1063783410110144, URL http://dx.doi.org/10.1134/S1063783410110144.

10 S. Zaitsev, M. Dorokhin, A. Brichkin, O. Vikhrova, Y. Danilov, B. Zvonkov, and V. Kulakovskii, JETP Letters 90, 658 (2010), ISSN 0021-3640, 10.1134/S0021364009220056, URL http://dx.doi.org/10.1134/S0021364009220056.

11 J. Schneider, U. Kaufmann, W. Wilkening, M. Baeumler, and F. Köhl, Phys. Rev. Lett. 59, 240 (1987), URL http://link.aps.org/doi/10.1103/PhysRevLett.59.240.

12 N. S. Averkiev and S. Y. Il'inskii, Phys. Solid. State 36, 278 (1994).

13 J. Bardeen, Phys. Rev. Lett. 6, 57 (1961), URL http://link.aps.org/doi/10.1103/PhysRevLett.6.57

14 W. Schairer and M. Schmidt, Phys. Rev. B 10, 2501 (1974), URL http://link.aps.org/doi/10.1103/PhysRevB.10.2501

15 V. F. Sapega, M. Ramsteiner, O. Brandt, L. Däweritz, and K. H. Ploog, Phys. Rev. B 73, 235208 (2006), URL http://link.aps.org/doi/10.1103/PhysRevB.73.235208.

16 T. Jungwirth, J. Sinova, J. Mašek, J. Kučera, and A. H. MacDonald, Rev. Mod. Phys. 78, 809 (2006), URL http://link.aps.org/doi/10.1103/RevModPhys.78.809.

17 D. A. Woodbury and J. S. Blakemore, Phys. Rev. B $\quad \mathbf{8}$ 3803 (1973), URL http://link.aps.org/doi/10.1103/PhysRevB.8.3803 